RADIONUCLIDE GUIDELINES

\title{
Procedure guidelines for radionuclide myocardial perfusion imaging
}

\author{
C Anagnostopoulos, M Harbinson, A Kelion, K Kundley, C Y Loong, A Notghi, E Reyes, W Tindale, \\ S R Underwood
}

Heart 2004;90(Suppl I):i1-i10

\section{BACKGROUND}

Radionuclide myocardial perfusion imaging uses an intravenous radiopharmaceutical to image myocardial perfusion during stimulation of the perfusion system and at rest. The images are acquired using a gamma camera. Tomographic imaging is preferred over planar imaging because of the three dimensional nature of the images and their superior contrast resolution. Comparison of the myocardial distribution of the radiopharmaceutical after stress and at rest provides information on myocardial viability, inducible perfusion abnormalities, and, when ECG gated imaging is used, global and regional myocardial function.

Radionuclide myocardial perfusion imaging is an established and non-invasive imaging technique with diagnostic and prognostic efficacy in the investigation of coronary artery disease. It is the only widely available test for assessing myocardial perfusion directly, but there are variations in the way it is performed in different centres. Harmonisation of practice, at least at a national level, is therefore essential, and clinical governance now makes it mandatory for practice to be based upon evidence whenever possible. ${ }^{1}$ This is best achieved by expert analysis of the evidence, and to this end the British Nuclear Cardiology Society (BNCS), in association with the British Cardiac Society (BCS) and the British Nuclear Medicine Society (BNMS), have developed procedure guidelines for tomographic myocardial perfusion imaging. The guidelines are intended to assist medical practitioners and other healthcare professionals in recommending, performing, interpreting, and reporting single photon emission computed tomography (SPECT) of myocardial perfusion. They do not cover the benefits or drawbacks of the technique in specific circumstances; neither do they address its cost-effectiveness in clinical diagnosis and management or its potential impact on clinical outcomes.

\section{METHODS OF GUIDELINE DEVELOPMENT}

The writing group was composed of clinicians and scientists from different specialties but all with subspecialty expertise in nuclear cardiology. The advisory group consisted of nominated representatives of the BNCS, the BNMS, and the guidelines committee of the BCS. Every effort was made to avoid conflict of interest from nonclinical relationships, and the final document was approved by the three societies.
A systematic search of Pub Med/Medline ${ }^{2}$ from January 1980 to June 2002 was undertaken. SPECT imaging was cross referenced with the following terms to find relevant articles: coronary artery disease, exercise and pharmacological stress, myocardial perfusion radiopharmaceuticals, attenuation correction, artefacts, ECG gating. Searches were limited to English language reports. In addition, previous published guidelines were reviewed (ACC/AHA exercise testing guidelines, ${ }^{3}{ }^{4}$ ACC/AHA/ACP-ASIM guidelines for the management of patients with chronic stable angina, ${ }^{5}$ American Society of Nuclear Cardiology imaging guidelines for nuclear cardiology procedures, ${ }^{6}$ and Society of Nuclear Medicine procedure guideline for myocardial perfusion imaging $\left.2.0^{7}\right)$. The search yielded 350 references, and those judged most representative are included in this document. Relevant data were summarised by each guideline developer and discrepancies reconciled by consensus. All recommendations are therefore based on evidence from clinical studies, previous published guidelines, or expert consensus of the writing and advisory groups.

\section{INDICATIONS FOR RADIONUCLIDE MYOCARDIAL PERFUSION IMAGING}

The indications are as follows.

- To assess the presence and degree of coronary obstruction in patients with suspected coronary artery disease

- To aid the management of patients with known coronary disease:

- to determine the likelihood of future coronary events, for instance after myocardial infarction or related to proposed non-cardiac surgery. ${ }^{8-10}$

- to guide strategies of myocardial revascularisation by determining the haemodynamic significance of coronary lesions. ${ }^{11}$

- to assess the adequacy of percutaneous and surgical revascularisation. ${ }^{12}$

- To assess myocardial viability and hibernation, particularly with reference to planned myocardial revascularisation. ${ }^{13}$

Abbreviations: ARSAC, Administration of Radioactive Substances Advisory Committee; BNCS, British Nuclear Cardiology Society; LBBB, left bundle branch block; LPO, left posterior oblique; RAO, right anterior oblique; SPECT, single photon emission computed tomography 
- Special indications are:

- To assess the haemodynamic significance of known or suspected anomalous coronary arteries and muscle bridging. ${ }^{14} 15$

- To assess the haemodynamic significance of coronary aneurysms in Kawasaki's disease. ${ }^{16} 17$

\section{STRESSING THE MYOCARDIAL PERFUSION SYSTEM}

4. 1 Dynamic exercise

4.1.1 Indication

Dynamic exercise is the stress technique of choice in the assessment of patients with suspected or known coronary artery disease, provided that the patient is able to exercise to an acceptable workload (for example, at least $85 \%$ of the maximum predicted heart rate). In particular, dynamic exercise is the ideal form of stress for patients with suspected or known anomalous coronary arteries, muscle bridging, or microvascular disease.

\subsubsection{Patient preparation}

- Withdrawal of drugs that may interfere with physiological exercise responses should be considered. In general, for the performance of diagnostic studies, $\beta$ adrenoceptor antagonists and rate limiting calcium channel antagonists should be discontinued for five half lives before the test unless medically contraindicated.

- Patients should also avoid caffeine containing foods, beverages, and drugs for a minimum of 12 hours before the test. This policy allows the use of vasodilator agents (dipyridamole, adenosine) in cases where the exercise is terminated and pharmacological stress is undertaken (see section 4.2 below).

- Patients should be instructed to dress appropriately for exercise.

- Fasting is not essential. While many centres routinely fast patients before imaging, the advantages of this policy are unproven.

\subsubsection{Protocol}

- Exercise testing must be undertaken by an appropriately trained healthcare professional. Guidelines for appropriate training are being developed by the BNCS but in the absence of these there must be a local statement of suitable training and experience. If the test is not being performed by a physician, a physician experienced in cardiovascular stress should be available for assistance with an urgency appropriate to the situation as defined in local or national guidelines. ${ }^{18}$

- The healthcare professional supervising the stress test should be up to date in immediate life support (ILS); there should be rapid access to personnel trained in advanced life support (ALS) and appropriate assistance and emergency support should be available.

- Initial evaluation should include a medical history (including symptoms, coronary risk factors, drug treatment, and previous diagnostic and therapeutic procedures) and a review of referral letters and other medical records if available. Physical examination may also be required, particularly if contraindications to dynamic exercise such as left ventricular outflow obstruction are suspected.

- Justification and authorisation for performing the test should be confirmed before starting, in compliance with current legislation.
- Dynamic exercise can be undertaken using a treadmill or a bicycle ergometer. Most treadmill protocols for exercise testing include an initial period of warm up, progressive uninterrupted exercise with increasing workload in each level until an end point is achieved, and a recovery period. The preferred method is the Bruce protocol. ${ }^{19}$ Bicycle ergometer protocols generally involve an initial low workload of 25 watts, followed by increases of 25 watts every two minutes until end points are achieved. ${ }^{3}$

- Regardless of the exercise protocol used, an intravenous line should be secured and flushed with $5-10 \mathrm{ml}$ of $0.9 \%$ sodium chloride injection to ensure patency before starting the test.

- Haemodynamic variables (heart rate and blood pressure) and ECG should be monitored at rest and throughout the test and recorded at each stage. Monitoring should continue for five minutes after exercise or until changes stabilise and haemodynamic variables and ECG are close to baseline. Monitoring with a 12 lead ECG is required for the detection of ST segment and T wave changes and for the diagnosis of arrhythmias.

- Exercise duration, symptoms, reason for stopping, and dynamic ECG changes should be noted.

\subsubsection{End points and radiopharmaceutical injection} Exercise should be symptom limited, with patients achieving at least $85 \%$ of the age and sex adjusted maximum predicted heart rate. The radiopharmaceutical should be injected close to peak exercise. The patient should continue exercising if feasible for one minute after thallium-201 injection, or for one to two minutes after technetium-99m perfusion tracer injection.

Exercise testing should be stopped if there is:

- ST segment elevation of more than $0.1 \mathrm{mV}$ in leads without Q waves;

- a fall in systolic blood pressure of more than $20 \mathrm{~mm} \mathrm{Hg}$ below baseline or of more than $20 \%$ from a previous stage despite an increase in workload, if this is considered to be related to myocardial ischaemia;

- hypertensive response (blood pressure $\geqslant 240 / 120 \mathrm{~mm} \mathrm{Hg}$ );

- serious arrhythmias (for example, ventricular fibrillation, ventricular tachycardia, frequent and symptomatic ventricular premature beats, multifocal ventricular premature beats, atrial fibrillation, supraventricular tachycardia, second or third degree atrioventricular block, and symptomatic bradycardia);

- severe angina;

- physical signs of peripheral hypoperfusion such as cyanosis or pallor;

- central nervous system symptoms such as ataxia, dizziness, or near syncope.

Horizontal or downsloping ST depression below baseline of $\geqslant 0.2 \mathrm{mV} 80 \mathrm{~ms}$ after the $\mathrm{J}$ point is not necessarily an indication for termination of exercise unless it is progressive or associated with symptoms.

\subsection{Pharmacological stress}

\subsubsection{Indication}

Pharmacological stress is an excellent alternative to dynamic exercise, provided that exercise tolerance, symptoms, and ECG changes during dynamic exercise are not required (table 1). It has the advantages of speed, reliability, and reproducibility, but the disadvantages that it is not possible to monitor the adequacy of stress and that it is not equivalent to the physiological stress experienced by the patient in 


\begin{tabular}{|c|c|c|}
\hline Agent & Protocol & Radiotracer injection time \\
\hline \multicolumn{3}{|l|}{ Vasodilators } \\
\hline Dipyridamole & $140 \mu \mathrm{g} / \mathrm{kg} / \mathrm{min}$ for $4 \mathrm{~min}$ & 4 min after completion of infusion \\
\hline Adenosine & $140 \mu \mathrm{g} / \mathrm{kg} / \mathrm{min}$ for $6 \mathrm{~min}$ & 3-4 min after start of infusion \\
\hline \multicolumn{3}{|l|}{ Inotropic agents } \\
\hline Dobutamine & $\begin{array}{l}\text { Starting at } 5-10 \mu \mathrm{g} / \mathrm{kg} / \mathrm{min} \text { and increasing } \\
\text { by } 5-10 \mu \mathrm{g} / \mathrm{kg} / \mathrm{min} \text { every } 3-5 \mathrm{~min} \text { up to } \\
40 \mu \mathrm{g} / \mathrm{kg} / \mathrm{min}\end{array}$ & $\begin{array}{l}\text { Peak stress ( } \geqslant 85 \% \mathrm{MPHR} \text { ) and } / \text { or } \\
\text { maximal dose }(40 \mu \mathrm{g} / \mathrm{kg} / \mathrm{min})\end{array}$ \\
\hline
\end{tabular}

everyday life. Pharmacological stress with vasodilators is the procedure of choice for patients unable to exercise adequately ${ }^{21}$ and for those with left bundle branch block (LBBB) or paced rhythm..$^{23}$

\subsubsection{Patient preparation Vasodilator stress}

Patients stressed with the vasodilators adenosine or dipyridamole must abstain from caffeine containing foods, beverages, and drugs for a minimum of 12 hours before the test and preferably for 24 hours. ${ }^{24} 25$ Aminophylline and theophylline must be stopped 24 hours before the test. ${ }^{26}$ Patients on dipyridamole should discontinue the drug for a minimum of 24 hours before vasodilator stress. A detailed explanation of the procedure should be given, outlining possible adverse effects and complications.

\section{Dobutamine stress}

Patients should stop $\beta$ adrenoceptor antagonists for five half lives or at least 24 hours before the test unless contraindicated. ${ }^{27} \mathrm{~A}$ detailed explanation of the procedure should be given, outlining possible adverse effects and complications.

\subsubsection{Protocol}

The stress must be undertaken by a suitably qualified healthcare professional as for dynamic exercise (see paragraph 4.1.3). Initial evaluation of the patient's medical history, examination if appropriate, and justification and authorisation for performing the test are mandatory.

\section{Adenosine stress}

For administration of adenosine, an intravenous line is required and a three way connector should be used to allow tracer injection without interruption of the adenosine infusion. However, the tracer injection should be given over 10 to 20 seconds to avoid a sudden bolus of adenosine. The adenosine is infused at $140 \mu \mathrm{g} / \mathrm{kg} /$ minute for six minutes using an infusion or syringe pump. This may be coupled with submaximal dynamic exercise when tolerated to reduce the frequency and severity of adverse effects associated with vasodilator infusion. ${ }^{28}$ If this is the case a bicycle ergometer is preferable to a treadmill because intravenous infusions are easily managed when the patient is relatively steady. Heart rate, blood pressure, and ECG should be measured and recorded at baseline and every two minutes during the infusion. The radiopharmaceutical is injected after three to four minutes of infusion or sooner if symptoms or other complications require. Tracer injection as early as two minutes after the start of the infusion is probably effective. Symptoms during the test should be recorded.

\section{Dipyridamole stress}

Intravenous dipyridamole is infused at a rate of $140 \mu \mathrm{g} / \mathrm{kg} /$ min for four minutes. The infusion can be given manually with care and it can be coupled with submaximal dynamic exercise when tolerated. Heart rate, blood pressure, and ECG should be measured and recorded at baseline and every two minutes during the infusion. The radiopharmaceutical should be injected four minutes after completion of the infusion. Symptoms during the test should be recorded. Dipyridamole causes adverse effects that are similar to those of adenosine, although they are generally more prolonged. ${ }^{29}$ Intravenous aminophylline (75-250 mg) may be required to reverse these effects, although its half life is shorter than that of dipyridamole (tables 2 and 3). ${ }^{30-34}$

\section{Dobutamine stress}

Dobutamine infusion is commonly used when dynamic exercise is not feasible and there are contraindications to vasodilator stress. It is given as an intravenous infusion using an infusion or syringe pump in three to five minute stages at incremental doses of $5,10,15,20,30$, and $40 \mu \mathrm{g} / \mathrm{kg} / \mathrm{min}^{32}{ }^{35}$ Heart rate and blood pressure should be recorded at the end of each stage and the ECG should be monitored continuously. Side effects may occur during infusion in up to $75 \%$ of patients (tables 2 and 3 ). The radiopharmaceutical should be injected when $\geqslant 85 \%$ of the age and sex adjusted maximum predicted heart rate is reached, or at $40 \mu \mathrm{g} / \mathrm{kg} / \mathrm{min}$, although stress may be adequate at lower heart rates. The dobutamine infusion should be continued for one minute after injection of thallium-201 or one to two minutes after injection of technetium-99m labelled tracers and is then stopped. Although atropine is given during dobutamine echocardiography if $85 \%$ of maximum predicted heart rate is not achieved, this may not be necessary for perfusion imaging because of the direct coronary dilating effect of dobutamine. ${ }^{36}{ }^{37}$ Dobutamine infusion should be discontinued for the same reasons as exercise testing (see paragraph 4.1.4).

\subsection{Precautions}

The presence of a healthcare professional who is up to date in ILS is required for the duration of all stress procedures. Personnel trained in ALS should be rapidly available. Emergency equipment, drugs, and support personnel should also be available.

Table 2 Percentages of reported side effects during pharmacological stress ${ }^{31-33}$

\begin{tabular}{llll}
\hline Side effects & $\begin{array}{c}\text { Dipyridamole } \\
\text { (n=391 1) }\end{array}$ & $\begin{array}{l}\text { Adenosine } \\
\text { (n=9256) }\end{array}$ & $\begin{array}{l}\text { Dobutamine } \\
\text { (n= 144) }\end{array}$ \\
\hline Chest pain & 20 & 35 & 31 \\
Dyspnoea & 3 & 35 & 14 \\
Flushing & 3 & 37 & 14 \\
Headache & 12 & 14 & 14 \\
Palpitations & 3 & 1 & 29 \\
Dizziness & 12 & 9 & - \\
Hypotension & 5 & 2 & 15 \\
High degree AV block & 2 & 5 & 0 \\
SVT/ventricular arrhythmias & 5 & 3 & 4 \\
Bronchospasm & 0.15 & 0.1 & 0 \\
\hline
\end{tabular}

$\mathrm{AV}$, atrioventricular; SVT, supraventricular tachycardia. 
Table 3 Summary of adverse events in patients undergoing stress for myocardial perfusion imaging ${ }^{30-34}$

\begin{tabular}{lllll}
\hline Event & $\begin{array}{l}\text { Exercise } \\
(\mathbf{n}=\mathbf{1 7 0 0 0 0 )}\end{array}$ & $\begin{array}{l}\text { Dobutamine } \\
(\mathbf{n}=\mathbf{3 0 1 1 )}\end{array}$ & $\begin{array}{l}\text { Dipyridamole } \\
(\mathbf{n}=\mathbf{3 9 1 1 )}\end{array}$ & $\begin{array}{l}\text { Adenosine } \\
(\mathbf{n}=\mathbf{9 2 5 6})\end{array}$ \\
\hline $\begin{array}{l}\text { Fatal Ml/cardiac death } \\
\text { Non-fatal Ml/major } \\
\text { cardiac complication }\end{array}$ & 0.01 & 0 & 0.05 & 0 \\
\hline
\end{tabular}

Values are percentages.

MI, myocardial infarction.

\subsection{Contraindications}

\subsubsection{Absolute contraindications to dynamic} exercise

- Non-ST-segment elevation acute coronary syndrome. Once stabilised, exercise stress can be considered 24 to 72 hours after chest pain, depending upon clinically assessed risk. ${ }^{38} 39$

- ST segment elevation myocardial infarction within the previous four days. ${ }^{40}$

- Left main coronary artery stenosis that is likely to be haemodynamically significant. ${ }^{3}$

- Left ventricular failure with symptoms at rest. ${ }^{3}$

- Recent history of life threatening arrhythmias. ${ }^{3}$

- Severe dynamic or fixed left ventricular outflow tract obstruction (aortic stenosis and obstructive hypertrophic cardiomyopathy). ${ }^{3}$

- Severe systemic hypertension (systolic blood pressure $>220$ $\mathrm{mm} \mathrm{Hg}$ and/or diastolic blood pressure $>120 \mathrm{~mm} \mathrm{Hg}){ }^{3}$

- Recent pulmonary embolism or infarction. ${ }^{3}$

- Thrombophlebitis or active deep vein thrombosis. ${ }^{3}$

- Active endocarditis, myocarditis, or pericarditis. ${ }^{3}$

\subsubsection{Relative contraindications to dynamic exercise}

- LBBB, bifascicular block, and ventricular paced rhythms, because dynamic exercise leads to perfusion abnormalities of the septum and adjacent walls in the absence of obstructive coronary disease. ${ }^{22} 23$

- Inability or poor motivation to perform dynamic exercise.

- Recent exercise ECG with inadequate exercise.

These are not strictly contraindications to dynamic exercise but they can compromise the accuracy of the test.

\subsubsection{Absolute contraindications to vasodilator stress}

- Recent acute coronary syndrome. Once stabilised, stress with vasodilators can be considered 24 to 72 hours after chest pain, depending upon clinically assessed risk. ${ }^{38-40}$

- Suspected or known severe bronchospasm. ${ }^{6}$

- Second and third degree atrioventricular block in the absence of a functioning pacemaker. ${ }^{6}$

- Sick sinus syndrome in the absence of a functioning pacemaker. $^{6}$

- Hypotension (systolic blood pressure $<90 \mathrm{~mm} \mathrm{Hg}$ ).

- Xanthines intake in the last 12 hours, or dipyridamole use in the last 24 hours. ${ }^{6}$

\subsubsection{Relative contraindications to vasodilator stress}

- Bradycardia of less than 40 beats/min. Initial dynamic exercise normally increases the rate sufficiently to start the infusion. ${ }^{6}$

- Recent cerebral ischaemia or infarction.

\subsubsection{Absolute contraindications to dobutamine stress}

- As for dynamic exercise above.

- Known hypokalaemia. ${ }^{41}$

\subsubsection{Relative contraindications to dobutamine stress}

- LBBB, bifascicular block, and paced rhythm, for the same reason as for dynamic exercise.

\section{RADIOPHARMACEUTICALS}

Thallium-201 and two technetium-99 $\mathrm{m}$ labelled radiopharmaceuticals (MIBI and tetrofosmin) are available commercially.

\subsection{Thallium-201}

Thallium-201 is initially distributed after intravenous injection to the myocardium according to myocardial viability and perfusion. It redistributes from this distribution over several hours, thus allowing redistribution images to be acquired that are independent of perfusion and reflect viability alone.

\subsubsection{Allowable activity}

The diagnostic reference level is $80 \mathrm{MBq}$ for stress and redistribution imaging. An additional injection of $40 \mathrm{MBq}$ can be given at rest for reinjection imaging if redistribution is thought to be incomplete at the time of redistribution imaging or if redistribution is predicted to be slow. ${ }^{42} 43$ Such reinjection activities are not normally approved as a routine by the United Kingdom Department of Health's Administration of Radioactive Substances Advisory Committee (ARSAC) $^{44}$ and must be given at the discretion of the practitioner in individual cases. A higher activity can be considered on an individual basis in obese patients.

\subsubsection{Administration}

- Thallium-201 should be given through a secure intravenous line in accordance with local radiation protection practices. If it is given through the side arm of a three way tap through which adenosine or dobutamine are running, it should be injected over 10-20 seconds to avoid a bolus of the pharmacological stressor being pushed ahead of the thallium-201. Otherwise it can be given as a bolus injection. The thallium-201 syringe can be flushed with three or four $0.5 \mathrm{ml}$ aliquots of either saline or the stressor solution to ensure that the full activity is given.

- If a resting injection is given, for instance in a patient with a severe defect of uptake in the stress images, sublingual glyceryl trinitrate $(400-800 \mu \mathrm{g})$ can be given at least five minutes beforehand in order to reduce resting hypoperfusion and to detect myocardial viability more accurately. Other nitrates such as buccal isosorbide dinitrate may also be used and these should be given in the supine position to avoid symptomatic hypotension. 


\subsubsection{Imaging protocols}

Different imaging protocols can be followed, depending on clinical indications and local practices: stress-redistribution, stress-reinjection, stress-redistribution-reinjection, or stressreinjection-delayed 24 hour imaging. ${ }^{45}$

Stress imaging should begin within five minutes of stress injection and should be completed within 30 minutes of injection.

Redistribution imaging should be done three to four hours after the stress injection.

In patients with severe perfusion defects in the stress images, or if redistribution is thought to be incomplete at the time of redistribution imaging, a resting injection can be given (ideally after sublingual nitrates) with reinjection imaging after a further 60 minutes of redistribution. ${ }^{46}$ This protocol is normally sufficient for the assessment of myocardial viability.

Imaging can also be done 24 hours after injection, using a longer acquisition time for the assessment of myocardial viability.

\subsection{Technetium-99 m MIBI and tetrofosmin}

After intravenous injection these technetium-99 m labelled radiopharmaceuticals are distributed within the myocardium according to myocardial viability and perfusion. Unlike thallium-201 they have minimal redistribution and so separate injections are required for stress and rest studies. The higher energy of technetium-99 m generally leads to better quality images (because of reduced attenuation and scatter) and permits ECG gating, which gives additional functional information. However, their uptake as a function of myocardial perfusion is less avid than thallium-201 and so defects may be less profound.

\subsubsection{Allowable activity}

The diagnostic reference level for tomography is a total of $1000 \mathrm{MBq}$ for a one day protocol (normally divided as 250 $\mathrm{MBq}$ and $750 \mathrm{MBq}$ ), or $400 \mathrm{MBq}$ for each study of a two day protocol. ${ }^{44}$ Higher activities can be considered on an individual basis by the practitioner, for instance in obese patients.

\subsubsection{Administration}

- The radiopharmaceutical should be given through a secure intravenous line in accordance with local radiation protection practices. The same considerations as described above for thallium-201 apply.

- As with thallium-201, resting injections can be given under nitrate cover and this is important when assessing myocardial viability because the absence of redistribution means that viability is underestimated in areas with reduced resting perfusion. ${ }^{47} 48$

\subsubsection{Imaging protocols}

Different imaging protocols can be followed, depending on clinical indications and local practices: one day stress-rest, one day rest-stress, or two day (especially for obese patients). The two day protocol is ideal from the imaging point of view but it may be less convenient for the patient. The one day protocols are acceptable alternatives.

Imaging should begin 30-60 minutes after injection to allow for hepatobiliary clearance, with longer delays required for resting images and for stress with vasodilators alone because of the higher liver uptake.

A fatty meal is given in some centres between injection and imaging to aid clearance of tracer from the liver and gall bladder. The value of this manoeuvre is uncertain and it may be counterproductive if there is retrograde passage of tracer from the duodenum to the stomach or if the tracer reaches the transverse colon. ${ }^{49} 50$

\section{IMAGE ACQUISITION}

Image acquisition should be done using a gamma camera that meets accepted standards of quality control. ${ }^{51}$

\section{1 Patient positioning}

The patient should be supine with both arms above the head and supported in a comfortable position. Knee support is also helpful and patient comfort is essential to minimise motion. Prone imaging has been used in some centres to reduce the incidence of inferior attenuation artefact ${ }^{52}$ but it can produce anterior artefacts and it is not recommended in isolation.

Female patients should be imaged without underclothes. A chest band can be used to minimise breast attenuation and to ensure reproducible positioning during later image acquisition. This can, however, increase attenuation depending upon how the band is applied, and careful attention to technique is required when the breasts are strapped. ${ }^{53}$ Chest bands can also be used in male subjects to reduce motion.

\subsection{Acquisition parameters}

- Tomographic imaging with a single or dual head gamma camera is usually carried out over a $180^{\circ}$ rotation from RAO $45^{\circ}$ to LPO $45^{\circ}$. With a dual headed camera the heads should ideally be at $90^{\circ}$ to each other for a $180^{\circ}$ rotation. A circular or non-circular orbit can be used according to preference. ${ }^{54}$

- Low energy general purpose collimation should be used for thallium-201 and high resolution collimation for technetium-99 $\mathrm{m}$ tracers. ${ }^{7}$

- A $15-20 \%$ energy window at 72 and $167 \mathrm{keV}$ for thallium201 and $140 \mathrm{keV}$ for technetium-99m labelled radiopharmaceuticals should be selected.

- The acquired pixel size should be in the region of $6 \mathrm{~mm}{ }^{6}$ A zoomed acquisition can be used depending upon camera dimensions, but this should be done carefully so that the patient lies within the field of view in all projections.

- A step and shoot acquisition with 32 or 64 stops separated by $3^{\circ}$ to $6^{\circ}$ or a continuous acquisition can be used. The duration of acquisition at each stop depends partly on the protocol, the activity of the radiopharmaceutical, and the patient's size. Typically, for a $90^{\circ}$ dual headed camera acquiring 64 images ( 32 projections for each detector), the time per projection would be 20 seconds for stress thallium-201, 25 seconds for redistribution thallium-201, 25 seconds for $250 \mathrm{MBq}$ stress technetium-99m acquisition, and 20 seconds for a non-gated $750 \mathrm{MBq}$ rest acquisition. Total acquisition times of longer than 20 to 30 minutes can be counterproductive as they increase the likelihood of patient motion. ${ }^{6}$

- ECG gating can be done, particularly with technetium$99 \mathrm{~m}$ labelled radiopharmaceuticals. Sixteen frames per cardiac cycle should be acquired for accurate calculation of the left ventricular ejection fraction. ${ }^{556}$

- Planar images can be acquired before the tomographic acquisition to determine the lung to heart ratio, although qualitative or quantitative assessment of lung/heart ratios can be made from the tomographic acquisition. ${ }^{7}$

- The planar projection images should be reviewed immediately after acquisition to check for unacceptable motion or other sources of artefact such as foreign objects or motion of the heart outside the field of view in some projections. $^{7}$ 


\section{IMAGE PROCESSING}

\subsection{Reconstruction}

Filtered back-projection using Butterworth and Hanning filters is the most common method of reconstruction. Cut off frequencies as per the manufacturer's recommendations-for example, 0.5 cycles/cm (order 5 or 10) and 0.75 cycles/cm, respectively_can be chosen, and these should be the same for each patient and should not be altered to compensate for low count images in order to maintain consistency of appearance. ${ }^{6}$

Iterative reconstruction is preferred if attenuation correction has been undertaken, and it can also be used without attenuation correction.

\subsection{Reorientation}

The long axis of the left ventricle is defined from the apex to the centre of the mitral valve, and definition of the axis can be manual or automatic. Automatic definitions should be checked and adjusted if necessary. The definition should be consistent in both stress and rest studies, bearing in mind that the orientation of the ventricle may change slightly between acquisitions.

The transverse tomograms are reoriented into three sets of oblique tomograms: (1) short axis (perpendicular to the long axis of the left ventricle); (2) vertical long axis (parallel to the long axis of the left ventricle and to the septum); and (3) horizontal long axis (parallel to the long axis of the left ventricle and perpendicular to the septum).

\subsection{Image evaluation}

The planar projection images and the reconstructed tomograms should be inspected immediately after acquisition by an operator or practitioner in order to identify technical problems that might require repeat acquisition. These might include:

- injection site or external objects passing across the heart;

- patient motion;

- inaccurate ECG gating;

- problems related to the detector such as drift in energy window and artefacts generated by transition between the two detectors;

- inappropriate collimation or energy windows.

\subsection{Image display}

Stress and rest images should be appropriately aligned and presented in a format that allows ready comparison of corresponding tomograms, such as interactive displays that triangulate the three planes or display the full set of tomograms.

Each tomographic acquisition should be displayed with the top of the colour scale at the maximum within the myocardium for each set. Displays with the top of the colour scale at the maximum of each individual tomogram and those that use the same maximum for stress and rest images should not be used. Care should be taken if the maximum lies outside the myocardium, and manual adjustment or masking of extracardiac activity may be required. The bottom end of the colour scale should be set to zero and background subtraction should be avoided. Neighbouring pairs of tomograms can be summed for display according to local preference. $^{57}$

\subsection{Attenuation correction}

Various techniques have been developed for correcting emission tomograms for attenuation in an effort to reduce or eliminate attenuation artefact. Many of these incorporate additional corrections for scatter and for depth dependent resolution recovery. Although initial results are encouraging, ${ }^{58}$ each method behaves differently and none overcomes artefacts entirely, some even introducing new forms of artefact from overcorrection..$^{59}$ The effectiveness of these techniques in routine clinical practice is currently uncertain. ${ }^{60}$ They should be used only in experienced centres and preferably as part of a formal assessment of their value. Corrected images should not be used without review alongside the uncorrected images.

\section{IMAGE INTERPRETATION}

\subsection{Review of clinical details}

It can be helpful initially to review the images without reference to clinical information-in order to decide upon major features-and then to modify the opinion and decide upon minor features if necessary after reviewing the clinical information. Attention should be paid to the patient's height, weight, and chest size-as these may influence the degree of attenuation and the quality of the study-and also to what findings would be expected from the clinical information. Unexpected findings are more likely to be artefactual.

The adequacy of stress should be noted, as well as the exercise time, symptoms, haemodynamic observations, and ECG changes. It is helpful to report in the company of the individuals who took the history, stressed the patient, and acquired the images, as symptoms and other observations during stress can influence reporting.

\subsection{Review of planar projection data}

Before interpreting the tomograms, the stress and rest planar projection data should be inspected alongside each other in a synchronised cine display using a linear grey scale (table 4) for the following reasons:

- to check that the heart is in the field of view throughout the acquisition;

- to look for sources of artefact including patient motion, upward creep, attenuation by soft tissue and external objects, hot activity adjacent to the heart that might obscure myocardial activity or cause reconstruction artefact, and low count artefact ${ }^{61}$;

- to look for evidence of left ventricular dilatation (either permanent or transient) or right ventricular hypertrophy or dilatation;

- to check whether there is increased lung uptake of tracer particularly thallium-201 (>50\% of maximum myocardial uptake), ${ }^{62}$ significant tracer uptake outside the heart, or extravasated radiopharmaceutical at the site of venepuncture;

- to assess the pattern of myocardial uptake, although this is seen more clearly in the tomograms.

\subsection{Review of tomograms}

8.3.1 Tomogram display

- Reconstructed tomograms should be viewed on a computer screen for reporting. Reporting from film or paper reproductions should be avoided.

Table 4 Sources of artefact apparent on cine review of projection images

Patient motion: craniocaudal and lateral

Upward creep

Attenuation by soft tissue (diaphragm and breast)

External objects

High activity adjacent to the heart

Low count density 
- The three tomographic planes should be displayed: vertical long axis, horizontal long axis, and short axis.

- A continuous colour scale should be used because it provides the best interobserver agreement. ${ }^{63}$

- For ECG gated and ungated studies, if automatic edge detection is used the computer derived edges should be inspected to ensure that they have been correctly defined. Incorrectly defined endocardial and epicardial borders will lead to wrong volume and ejection fraction calculations, and to incorrect polar displays and quantification. ${ }^{55}$

\subsubsection{Left ventricular size and right ventricular uptake and size}

Assessment of the tomographic images should begin with a qualitative assessment of the left ventricular cavity size in both sets of images. Dilatation that is worse in the stress images than at rest indicates ischaemia induced dilatation. ${ }^{62}$ This is seen less commonly with technetium-99 m tracers because of the delayed imaging. Care should be taken that areas of reduced uptake in the stress images do not simulate dilatation.

Tracer uptake in the right ventricle should also be noted. Significant right ventricular tracer uptake $(>50 \%$ of maximum left ventricular uptake) indicates right ventricular hypertrophy, and the right ventricle may also be dilated. ${ }^{64}$

\subsubsection{Perfusion defect localisation, extent and severity}

Tracer uptake should be evaluated visually in all areas of the left ventricular myocardium. Segmental analysis can be done using various models of the left ventricular myocardium, and a 17 segment model is recommended by several American societies. ${ }^{65}$

Tracer uptake can be classified semiquantitatively as normal (100-70\% maximum uptake), mildly reduced (69$50 \%$ maximum uptake), moderately reduced (49-30\% maximum uptake), severely reduced (29-10\% maximum uptake), and absent (9-0\% maximum uptake). These figures are approximate and allowance should be made for normal variation and for artefact. Thus the inferior wall may be judged to have normal uptake at much lower values if attenuation artefact is considered to be present. ${ }^{66}$

\subsubsection{Review of ECG gated tomograms}

- The beat length histogram, if available, and the timevolume curve should be inspected to ensure that gating was appropriate. Cine inspection of the gated tomograms may also give clues of inadequate gating, such as inappropriate positioning of diastole or reduced counts in some frames.

- The computer derived endocardial and epicardial edges should be checked to ensure that they have been appropriately selected.

- Wall motion is best evaluated in linear grey scale without computer derived edges, and can be classified as normal, hypokinetic, akinetic, or dyskinetic (paradoxical). ${ }^{67}$ Computer generated contours can be helpful but these should not be used as the sole determinant of motion.

- Wall thickening is best evaluated in a continuous colour scale without computer derived edges, and is related to the increase in counts between diastole and systole. Computer generated contours can be helpful but these should not be used as the sole determinant of thickening. Thickening can be classified as normal, reduced, or absent. ${ }^{67}$

- Left ventricular end diastolic volume, end systolic volume, stroke volume, and ejection fraction may be calculated automatically, although the values obtained should be checked against initial qualitative assessment. Caution should be exercised in reporting apparently spurious values of these variables. For instance, volumes are often too low and ejection fraction too high in small ventricles. ${ }^{55}$

\subsection{Quantification}

For routine clinical reporting, formal quantitative analysis may not be necessary. However, it can be helpful to supplement semiquantitative visual analysis with quantitative analysis of the polar display, particularly to measure the extent and depth of abnormalities. ${ }^{68}$ The patient's polar map is compared with a normal database, which should be sex and radionuclide specific and may also be institute specific. ${ }^{55}$ An alternative to the polar display is the display of circumferential count profiles, but this is less common. Any form of quantification should be validated in published studies, and the methodology used should be fully described and understood by those who use the technique. Quantitative results must not be reported in isolation and without expert review of the images from which the results are derived.

\subsection{Integration of findings}

The tomographic findings should be integrated to reach a final interpretation:

- An improvement in relative tracer uptake from stress to rest ("inducible perfusion abnormality") often indicates the presence of inducible ischaemia. ${ }^{69}$ An improvement in tracer uptake of one category indicates mild inducible ischaemia, of two categories moderate inducible ischaemia, and of more than two categories severe inducible ischaemia.

- A reduction in tracer uptake that does not change from stress to rest ("fixed perfusion abnormality") normally indicates myocardial infarction, and the degree of reduction indicates the transmural extent of infarction from mild partial thickness infarction to full thickness infarction.

- Differentiation between true abnormality of tracer uptake and artefact requires experience. Features in favour of attenuation artefact are visualisation of the attenuating structure in the projection images, the fixed nature of the defect (especially if moving normally on ECG gated images), an expected site (for example, inferior wall or anterior wall in women) of limited extent, smooth edges, poor correspondence with a coronary territory, or an unexpected finding. None of these features, however, is universally reliable. Features indicating reconstruction artefact are a limited mild to moderate fixed defect at the apex ("apical thinning") or intense liver or gall bladder activity that passes behind the inferior wall in the projection images. ${ }^{61}$

- A deterioration in tracer uptake from stress to rest ("rapid tracer washout" or "reverse redistribution") is often artefactual but may suggest partial thickness infarction with a patent artery. ${ }^{70}$

\subsection{Reporting}

\subsubsection{Patient details}

The patient's personal details (name, age, sex and address) should be included at the start of the report. Any hospital/ clinic identification number and source of referral should also be included (table 5).

\subsubsection{Type of study}

The imaging protocol should be specified, including the radiopharmaceutical used, imaging technique, sequence, and date of study. 


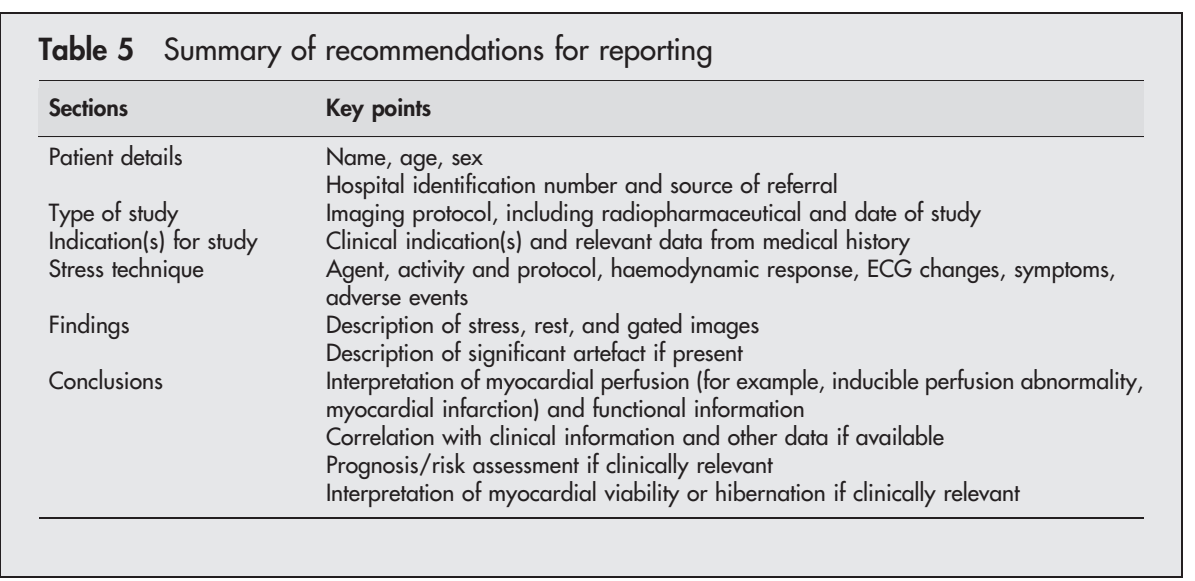

\subsubsection{Indications for study}

The clinical indications for the study should be stated, including any relevant clinical history. This provides justification for the study, summarises clinical information that may have been gleaned from a number of sources, and focuses the final conclusion.

\subsubsection{Stress technique}

The stress technique used should be described briefly, including any symptoms, haemodynamic changes, and details of ECG changes during or after stress if relevant.

\subsubsection{Findings}

The appearance of the stress, rest, and gated images should be described succinctly, including a statement on overall study quality if appropriate. Common practice is to report the defects in the stress tomograms in decreasing order of severity, and then to state how each defect changes in the rest tomograms in the same order. At this stage tracer uptake is being described. Clinical deductions such as the state of myocardial viability and perfusion can be reserved for the conclusions (see below).

\subsubsection{Conclusions}

- The findings should be integrated to reach a final interpretation. ${ }^{66}$ Specifically, the report should comment on the presence (if any) of inducible perfusion abnormality, infarction, and significant artefact. If there is an abnormality, its location (in terms of segments affected), extent (in terms of number of segments affected), and severity should be stated.
- Other abnormalities to mention, if present, are left ventricular dilatation (persistent or transient), increased lung uptake of tracer, right ventricular tracer uptake suggesting hypertrophy (with or without right ventricular dilatation), and significant non-cardiopulmonary tracer uptake.

- If the study is normal, this should be stated specifically, bearing in mind that homogeneous myocardial perfusion during stress does not exclude non-obstructive coronary disease.

- A statement on the likelihood of future coronary events should be made if clinically relevant. This is deduced from the presence, extent, and depth of inducible perfusion abnormalities, the left ventricular ejection fraction if known, and other markers of prognosis such as transient dilatation and lung uptake. If no inducible perfusion abnormalities are present then the ejection fraction is the main determinant of prognosis. This statement should ideally be made in semiquantitative terms (for example, "the likelihood of future coronary events is in the region of $5-10 \%$ per year") as qualitative terms ("high", "intermediate", "low") are not uniformly interpreted.

- If assessment of myocardial viability/hibernation is relevant, or correlation with coronary anatomy is required, these should be commented on bearing in mind the normal variation of coronary anatomy.

- Finally, it should be ensured that the conclusions answer the clinical question that prompted the referral if possible, and if not it may be relevant to make recommendations for further investigation or management.

Table 6 Factors affecting the quality of studies

\begin{tabular}{ll}
\hline Phase of study & Factor \\
\hline Stress & $\begin{array}{l}\text { Incorrect agent or protocol } \\
\text { Submaximal stress }\end{array}$ \\
Drug treatment-for example, use of antagonists (if the study is done for \\
diagnostic purposes) \\
Inadequate activity for patient weight/size \\
Misadministration (for example, extravascular injection) \\
Image acquisition & $\begin{array}{l}\text { Inadequate camera positioning or orbit selection } \\
\text { Inappropriate energy window selection or collimation } \\
\text { Patient comfort and motion } \\
\text { External attenuating objects or inadequate breast strapping } \\
\text { Incorrect ECG gating } \\
\text { Inappropriate filtering or reconstruction technique } \\
\text { Inaccurate definition of long axis of left ventricle } \\
\text { Inappropriate colour/grey scale or incorrect windowing } \\
\text { Comparison of non-equivalent tomograms }\end{array}$ \\
\hline
\end{tabular}




\section{FACTORS AFFECTING THE QUALITY OF STUDIES 9.1 Stress technique}

Inadequate stress reduces the sensitivity for detecting coronary artery disease (table 6). ${ }^{20} 72$

\subsection{Tracer activity and delivery}

Inadequate delivery of the radiopharmaceutical degrades image quality and may decrease the diagnostic accuracy of the technique. This may occur if the wrong activity of tracer for patient weight/size is given or if the injection is inadequately flushed or extravasated.

Inappropriately timed tracer delivery (that is, not coinciding with peak stress) may reduce the sensitivity of the technique.

\subsection{Image reconstruction and processing}

Inappropriate filtering during tomographic reconstruction may degrade image quality, while inappropriate use of colour or grey scale windows may lead to diagnostic inaccuracies. For quantitative analysis of regional myocardial and lung activity, care should be taken that regions of interest do not include activity from adjacent structures. ${ }^{73}$

Advisory Group: A van Aswegen, A J W Hilson, M Metcalfe, T Nunan, M Prescott, E M Prvulovich, A C Tweddel, G Vivian

Adopted by the British Cardiac Society, the British Nuclear Cardiology Society, and the British Nuclear Medicine Society

\section{REFERENCES}

1 Secretary of State for Health. The new NHS. London: HMSO, $1997(\mathrm{Cm}$ 3807).

2 Entrez-PubMed. [Homepage of the PuBMed service of the United States National Library of Medicine, May 2002] [online]. Available from URL: http://www.ncbi.nlm.nih.gov/entrez/query.fcgi.

3 Gibbons RJ, Balady GJ, Beasley J, et al. ACC/AHA guidelines for exercise testing. J Am Coll Cardiol 1997;30:260-311

4 Gibbons RJ, Balady GJ, Bricker TJ, et al. ACC/AHA 2002 guideline update for exercise testing: summary article. A report of the American College of Cardiology/American Heart Association task force on practice guidelines. Circulation 2002:106:1883-92.

5 Gibbons RJ, Chatteriee K, Daley J, et al. ACC/AHA/ACP-ASIM guidelines for the management of patients with chronic stable angina: a report of the American College of Cardiology/American Heart Association task force on practice guidelines. J Am Coll Cardiol 1999;33:2092-7.

6 DePuey EG, Garcia E, Borges-Neto S, et al. Updated imaging guidelines for nuclear cardiology procedures, part 1. J Nucl Cardiol 2001;8:G1-58.

7 Strauss HW, Miller DD, Wittry MD, et al. Procedure guidelines for myocardial perfusion imaging 2.0. Society of Nuclear Medicine procedure guidelines manual. 2001-2002.

8 Hachamovitch $\mathbf{R}$, Berman DS, Kiat $\mathrm{H}$, et al. Exercise myocardial perfusion SPECT in patients without known coronary artery disease: incremental prognostic value and use in risk stratification. Circulation 1996;93:905-14

9 Brown KA, Heller GV, Landin RS, et al. Early dipyridamole-(99m) Tc-sestamibi single- photon emission computed tomography imaging 2 to 4 days after acute myocardial infarction predicts in-hospital and post discharge cardiac events: comparison with sub maximal exercise imaging. Circulation 1999; 100:2060-6.

10 Eagle KA, Berger PB, Calkins $\mathrm{H}$, et al. ACC/AHA guideline update on perioperative cardiovascular evaluation for noncardiac surgery. Circulation 2002; 105: 1257-67

11 Heller LI, Cates C, Popma J, et al. Intracoronary Doppler assessment of moderate coronary artery disease. Comparison with thallium-201 imaging and coronary angiography. Circulation 1997:96:484-90.

12 Zellweger MJ, Lewin HC, Lai S, et al. When to stress patients after coronary artery bypass surgery? Risk stratification in patients early and late post-CABG using stress myocardial perfusion SPECT: implications of appropriate clinical strategies. J Am Coll Cardiol 2001;37:144-52.

13 Allman KC, Shaw $U$, Hachamovitch R, et al. Myocardial viability testing and impact of revascularisation on prognosis in patients with coronary artery disease and left ventricular dysfunction: a meta-analysis. J Am Coll Cardiol 2002;39:1 151-8.

14 Donaldson RM, Raphael M, Radley-Smith R, et al. Angiographic identification of primary coronary anomalies causing impaired myocardial perfusion. Cathet Cardiovasc Diagn 1983;9:237-49.

15 Lee YS, Moon DH, Shin JW, et al. Dipyridamole Tl-201 SPECT imaging in patients with myocardial bridging. Clin Nucl Med 1999;24:759-64.

16 Fukuda T, Akagi T, Ishibashi $M$, et al. Non-invasive evaluation of myocardial ischaemia in Kawasaki disease: comparison between dipyridamole stress thallium imaging and exercise stress testing. Am Heart J 1998;135:482-7.

17 Miyagawa M, Mochizuki T, Murase K, et al. Prognostic value of dipyridamole-thallium myocardial scintigraphy in patients with Kawasaki disease. Circulation 1998;98:990-6.
18 Rodgers G, Ayanian J, Balady G, et al. ACC/AHA Clinical competence statement on stress testing. Circulation 2000;102:1726-38.

19 Hill J, Timmis A. Exercise tolerance testing. BMJ 2002;324:1084-7

20 Iskandrian AS, Heo J, Kong B, et al. Effect of exercise level on the ability of thallium-201 tomographic imaging in detecting coronary artery disease: analysis of 461 patients. J Am Coll Cardiol 1989;14:1487-90.

21 Gupta NC, Esterbrooks DJ, Hilleman DE, et al. Comparison of adenosine and exercise thallium-201 single-photon emission computed tomography (SPECT) myocardial perfusion imaging. J Am Coll Cardiol 1992;19:248-57.

22 Tawarahara K, Kurata C, Tagushi T, et al. Exercise testing and thallium-201 emission computed tomography in patients with intraventricular conduction disturbances. Am J Cardiol 1992;69:97-102.

23 O'Keefe JH, Bateman TM, Barnhart CS. Adenosine thallium-201 is superior to exercise thallium-201 for detecting coronary artery disease in patients with left bundle branch block. J Am Coll Cardiol 1993;21:1332-8.

24 Smits P, Corstens FH, Aengevaeren WR, et al. False-negative dipyridamole thallium-201 myocardial perfusion imaging after caffeine infusion. J Nucl Med 1991;32:1538-41.

25 Böttcher M, Czernin J, Sun KT, et al. Effect of caffeine on myocardial blood flow at rest and during pharmacological vasodilation. J Nucl Med 1995;36:2016-21.

26 Smits $\mathbf{P}$, Lenders JW, Thien T. Caffeine and theophylline attenuate adenosineinduced vasodilation in humans. Clin Pharmacol Ther 1990:48:410-8.

27 Shehata AR, Gillam LD, Mascitelli VA, et al. Impact of acute propanolol administration on dobutamine-induced myocardial ischaemia as evaluated by myocardial perfusion imaging and echocardiography. Am J Cardiol 1997; 80:268-72

28 Pennell DJ, Mavrogeni SI, Forbat SM, et al. Adenosine combined with dynamic exercise for myocardial perfusion imaging. J Am Coll Cardiol 1995;25:1300-9

29 Taillefer R, Amyot R, Turpin S, et al. Comparison between dipyridamole and adenosine as pharmacological coronary vasodilators in detection of coronary artery disease with thallium-201 imaging. J Nucl Cardiol 1996;3:204-11.

30 Ranhosky A, Kempthorne-Rawson J. The safety of intravenous dipyridamole thallium myocardial perfusion imaging. Circulation 1990;81:1205-9.

31 Cerqueira M, Verani M, Schwaiger M, et al. Safety profile of adenosine stress perfusion imaging: results from the Adenoscan multicentre trial registry. J Am Coll Cardiol 1994;23:384-9.

32 Hays JT, Mahmarian JJ, Cochran AJ, et al. Dobutamine thallium-201 tomography for evaluating patients with suspected coronary disease unable to undergo exercise or vasodilator pharmacologic stress testing. J Am Coll Cardiol 1993;21:1583-90.

33 Rochmis P, Blackburn H. Exercise Tests. A survey of procedures, safety, and litigation experience in approximately 170,000 tests. JAMA 1971;217:1061-6.

34 Secknus MA, Marwick TH. Evolution of dobutamine echocardiography protocols and indications: safety and side effects in 3011 studies over 5 years. J Am Coll Cardiol 1997;29:1234-40

35 Geleijnse M, Elhendy A, Fioretti $P$, et al. Dobutamine stress myocardial perfusion imaging. J Am Coll Cardiol 2000;36:2017-27.

36 Dubois-Randé JL, Merlet P, Duval-Moulin AM, et al. Coronary vasodilating action of dobutamine in patients with idiopathic dilated cardiomyopathy. Am Heart J 1993; 125:1329-36.

37 Bartunek J, Wiins W, Heyndrickx GR, et al. Effects of dobutamine on coronary stenosis physiology and morphology. Comparison with intracoronary adenosine. Circulation 1999; 100:243-9.

38 British Cardiac Society Guidelines and Medical Practice Committee and Royal College of Physicians Clinical Effectiveness and Evaluation Unit (2001). Guidelines for the management of patients with acute coronary syndromes without persistent ECG ST segment elevation. Heart 2001;85:133-42.

39 Braunwald E, Antman EM, Beasley JW, et al. ACC/AHA 2000 guidelines for the management of patients with unstable angina and non-ST-segment elevation myocardial infarction. A report of the American College of Cardiology/American Heart Association task force on practice guidelines. J Am Coll Cardiol 2000;36:970-1062.

40 Ryan TJ, Antman EM, Brooks NH, et al. 1999 update: ACC/AHA guidelines for the management of patients with acute myocardial infarction. A report of the American College of Cardiology/American Heart Association task force on practice guidelines. J Am Coll Cardiol 1999;34:890-911

41 Coma-Canella I. Changes in plasma potassium during the dobutamine stress test. Int J Cardiol 1991;33:55-60.

42 Dilsizian V, Rocco TP, Freedman NM, et al. Enhanced detection of ischaemic but viable myocardium by the reinjection of thallium after stress-redistribution imaging. N Engl J Med 1990;323:141-6.

43 van Eck-Smit BL, van der Wall EE, Zwinderman $\mathrm{AH}$, et al. Clinical value of immediate thallium-201 reinjection imaging for the detection of ischaemic heart disease. Eur Heart J 1995; 16:410-20.

44 Administration of Radioactive Substances Advisory Committee (ARSAC) Diagnostic procedures - adult patients. 2002:37-9.

45 Hicks RJ. Myocardial perfusion scintigraphy techniques using single photon radiotracers. In: Murray E, ed. Nuclear medicine in diagnosis and treatment, 2nd ed. New York: Churchill Livingstone, 1998:1333-51

46 Rocco TP, Dilsizian V, McKusick KA, et al. Comparison of thallium redistribution with rest "reinjection" imaging for detection of viable myocardium. Am J Cardiol 1990;66:158-63.

47 Maurea S, Cuocolo A, Soricelli A, et al. Enhanced detection of viable myocardium by technetium- $99 \mathrm{~m}-\mathrm{M} \mid \mathrm{BI}$ imaging after nitrate administration in chronic coronary artery disease. J Nucl Med 1995;36:1945-52.

48 Thorley PJ, Bloomer TN, Sheard KL, et al. The use of GTN to improve the detection of ischaemic myocardium using Tc-99m-tetrofosmin. Nucl Med Commun 1996;17:669-74. 
49 van Dongen AJ, van Rijk PP. Minimizing liver, bowel, and gastric activity in myocardial perfusion SPECT. J Nucl Med 2000;41:1315-17.

50 Hurwitz GA, Clark EM, Slomka PJ, et al. Investigation of measures to reduce interfering abdominal activity on rest myocardial images with Tc-99m sestamibi. Clin Nucl Med 1993;18:735-41.

51 Groch MW, Erwin WD. Single-photon emission computed tomography in the year 2001: instrumentation and quality control. J Nucl Med Technol 2001;29:12-18.

52 Segall GM, Davis MJ. Prone versus supine thallium myocardial SPECT: a method to decrease artefactual inferior wall defects. J Nucl Med 1989;30:548-55.

53 Germano G. Technical aspects of myocardial SPECT imaging. J Nud Med 2001;42:1499-507.

54 Eisner RL, Nowak DJ, Pettigrew R, et al. Fundamentals of 180-degree acquisition and reconstruction in SPECT imaging. J Nucl Med 1986:27:1717-28.

55 Germano G, Berman DS. Quantitative gated perfusion SPECT. In: Germano G, Berman DS, eds. Clinical gated cardiac SPECT. New York: Futura, 1999: 115-46.

56 Germano G, Kiat H, Kavanagh PB, et al. Automatic quantification of ejection fraction from gated myocardial perfusion SPECT. J Nucl Med 1995;36:2138-47.

57 van Train K, Folks R, Wong C, et al. Optimization of Tc-MIBI SPEC acquisition and processing parameters: collimator, matrix size and filter evaluation [abstract]. J Nucl Med 1989;30:P757.

58 Ficaro EP, Fessler JA, Shreve PD, et al. Simultaneous transmission/emission myocardial perfusion tomography. Diagnostic accuracy of attenuation-

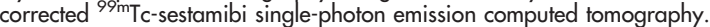
Circulation 1996;93:463-73.

59 O'Connor MK, Kemp B, Anstett F, et al. A multicenter evaluation of commercial attenuation compensation techniques in cardiac SPECT using phantom models. J Nucl Cardiol 2002:9:361-76.

60 Hendel RC, Corbett JR, Cullom SJ, et al. The value and practice of attenuation correction for myocardial perfusion SPECT imaging: a joint position statement from the American Society of Nuclear Cardiology and the Society of Nuclear Medicine. J Nucl Cardiol 2002;9:135-43.
61 DePuey EG. How to detect and avoid myocardial perfusion SPECT artifacts. J Nud Med 1994;53:699-702.

62 Iskandrian AS, Heo J, Nguyen T, et al. Left ventricular dilatation and pulmonary thallium uptake after single-proton emission computed tomography using thallium-201 during adenosine-induced coronary hyperemia. Am J Cardiol 1990;66:807-11.

63 Candell-Riera J, Santana-Boado C, Bermejo B, et al. Interhospital observer agreement in interpretation of exercise myocardial Tc-99m-tetrofosmin SPECT studies. J Nucl Cardiol $2001 ; 8: 49-57$.

64 Winzelberg GG. Right ventricular visualization-thallium-201 cardiac imaging. Semin Nucl Med 1982;12:301-3.

65 Cerqueira M, Weissman N, Dilsizian V, et al. Standardized myocardial segmentation and nomenclature for tomographic imaging of the heart. Circulation 2002; 105:539-42.

66 Pennell DJ, Prvulovich E. Image interpretation: nuclear cardiology. London: British Nuclear Medicine Society, 1995:56-79.

67 Berman DS, Germano G. An approach to the interpretation and reporting of gated myocardial perfusion SPECT. In: Germano G, Berman DS, eds. Clinical gated cardiac SPECT. New York: Futura, 1999:147-82.

68 Mahmarian JJ, Boyce TM, Goldberg RK, et al. Quantitative exercise thallium201 single photon emission computed tomography for the enhanced diagnosis of ischaemic heart disease. J Am Coll Cardiol 1990;15:318-29.

69 Pohost GM, Alpert NS, Ingwall JP, et al. Thallium redistribution: mechanisms and clinical utility. Semin Nucl Med 1980;20:70-93.

70 Weiss AT, Maddahi J, Lew AS, et al. Reverse redistribution of thallium-201: a sign of non transmural myocardial infarction with patency of the infarctrelated coronary artery. J Am Coll Cardiol 1986;7:61-7.

71 Araujo W, DePuey EG, Kamran M et al. Artefactual reverse redistribution pattern in myocardial perfusion SPECT with technetium-99m sestamibi. J Nucl Cardiol 2000;7:633-8.

72 Verziillbergen JF, Vermeersch PH, Laarman GJ, et al. Inadequate exercise leads to sub optimal imaging. Thallium-201 myocardial perfusion imaging after dipyridamole combined with low-level exercise unmasks ischaemia in symptomatic patients with non-diagnostic thallium-201 scans who exercise submaximally. J Nucl Med 1991;32:2071-8.

73 Hitzel A, Manrique A, Cribier A, et al. Diagnostic value of Tl-201 lung uptake is dependent on measurement method. J Nucl Cardiol 2001;8:332-8.

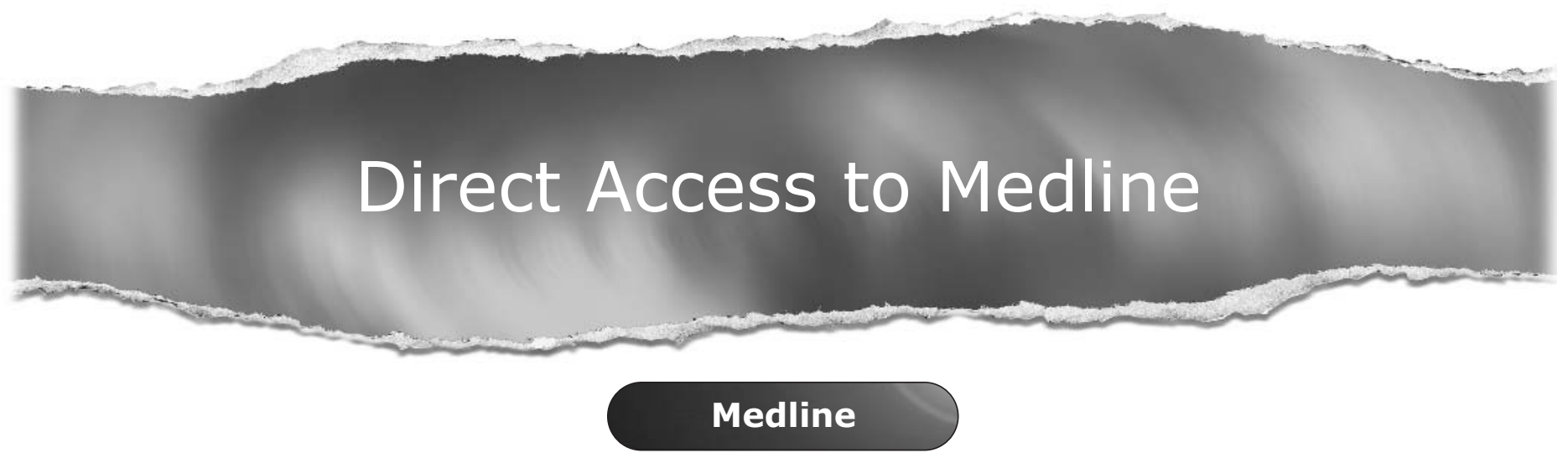

Link to Medline from the homepage and get straight into the National Library of Medicine's premier bibliographic database. Medline allows you to search across 9 million records of bibliographic citations and author abstracts from approximately 3,900 current biomedical journals.

\section{www.heartjnl.com}

